\title{
CONVERGENCE OF A HIGH-ORDER COMPACT FINITE DIFFERENCE SCHEME FOR A NONLINEAR BLACK-SCHOLES EQUATION
}

\author{
Bertram Düring ${ }^{1}$, Michel FourniÉ ${ }^{2}$ And Ansgar JÜngel ${ }^{1}$
}

\begin{abstract}
A high-order compact finite difference scheme for a fully nonlinear parabolic differential equation is analyzed. The equation arises in the modeling of option prices in financial markets with transaction costs. It is shown that the finite difference solution converges locally uniformly to the unique viscosity solution of the continuous equation. The proof is based on a careful study of the discretization matrices and on an abstract convergence result due to Barles and Souganides.
\end{abstract}

Mathematics Subject Classification. 49L25, 65M06, 65M12.

Received: January 13, 2004.

\section{INTRODUCTION}

In an idealized financial market the price of a European option can be obtained as the solution of the celebrated Black-Scholes equation [6,24]. This equation also provides a hedging portfolio that perfectly replicates the contingent claim. However, the Black-Scholes equation has been derived under quite restrictive assumptions (for instance, frictionless, liquid and complete markets). In recent years, some of these assumptions have been relaxed in order to model, for instance, the presence of transaction costs $[3,8,12]$, feedback effects due to large traders $[14-16,20,26,28]$, and incomplete markets [23]. In this paper we are concerned with the numerical discretization of a nonlinear Black-Scholes equation modeling transaction costs arising in the hedging of portfolios.

If transaction costs are taken into account perfect replication of the contingent claim is no longer possible, and it has been shown in [29] that further restrictions are needed in the model. A popular approach is to introduce preferences by assuming that the investor's behavior is characterized by a given utility function. In [17] it has been shown that the option price can be obtained as the cash increment which offsets the difference between the maximum utility of terminal wealth when there is no option liability and when there is such a liability. Davis et al. [12] extended this approach to markets with transaction costs (see also [3,32]). It has the disadvantage that the option price depends on the special choice of the utility function but Constantinides and Zariphopoulou [9] obtained universal bounds independent of the utility function.

Using this utility maximization approach, the following model has been proposed by Barles and Soner [3]. Assuming an exponential utility function $U(x)=1-\exp (-x / \varepsilon)$ with $\varepsilon=1 / \gamma N>0$, where $\gamma$ is the risk aversion factor and $N$ the number of options to be sold, they perform an asymptotic analysis in the limit $\varepsilon \rightarrow 0, \mu \rightarrow 0$

\footnotetext{
Keywords and phrases. High-order compact finite differences, numerical convergence, viscosity solution, financial derivatives.

1 Fachbereich Mathematik und Informatik, Johannes Gutenberg-Universität Mainz, Germany. e-mail: duering@uni-mainz.de

2 UMR-CNRS 5640, Laboratoire MIP, Université Paul Sabatier, Toulouse, France.
} 
such that $a=\mu / \sqrt{\varepsilon}$ is a constant transaction cost parameter, deriving the following nonlinear Black-Scholes equation for the option price $V(S, \tau)$

$$
V_{\tau}+\frac{1}{2} \sigma\left(V_{S S}\right)^{2} S^{2} V_{S S}+\rho S V_{S}-\rho V=0
$$

where the nonlinear volatility $\sigma\left(V_{S S}\right)$ is given by

$$
\sigma\left(V_{S S}\right)=\sigma_{0}\left(1+\Psi\left[\exp \left(\rho\left(\tau_{0}-\tau\right)\right) a^{2} S^{2} V_{S S}\right]\right) .
$$

Here, $\rho$ denotes the risk-free interest rate and $\tau_{0}$ the maturity. The function $\Psi$ is the solution of the nonlinear initial-value problem

$$
\Psi^{\prime}(A)=\frac{\Psi(A)+1}{2 \sqrt{A \Psi(A)}-A}, A \neq 0, \quad \Psi(0)=0 .
$$

Equation (1) is solved for the price $S \geq 0$ of the underlying asset and time $\tau_{0} \geq \tau \geq 0$, i.e. backward in time. The terminal condition is

$$
V\left(S, \tau_{0}\right)=V_{0}(S), \quad S \geq 0 .
$$

The equation is derived in [3] for European Call options, i.e. $V_{0}(S)=\max (0, S-E)$, where $E>0$ is the exercise price. The "boundary" conditions are as follows

$$
V(0, \tau)=0, \quad V(S, \tau) \sim S-E \mathrm{e}^{\rho\left(\tau-\tau_{0}\right)} \quad(S \rightarrow \infty), \quad \tau_{0} \geq \tau \geq 0 .
$$

The last condition has to be understood in the sense

$$
\lim _{S \rightarrow \infty} \frac{V(S, \tau)}{S-E \mathrm{e}^{\rho\left(\tau-\tau_{0}\right)}}=1,
$$

uniformly for $\tau_{0} \geq \tau \geq 0$. In [3] the existence of a unique continuous viscosity solution $V$ to this problem has been shown.

In [13] we discretized the nonlinear Black-Scholes equation (1) with volatility (2) and proposed a new highorder compact finite difference scheme generalizing a scheme of Rigal [27]. The nonlinearity was treated explicitly, i.e., the final scheme is semi-implicit. We studied the properties of the new compact scheme R3C and showed that the scheme is unconditionally stable (in the sense of von Neumann) and non-oscillatory. It turned out that it gives significantly better results than classical schemes. The compact scheme R3C combines good properties (stability, non-oscillations) with a high order of accuracy. It can be considered as more efficient since the relation between CPU time and $\ell_{2}$-error is better compared with classical schemes (see [13] for details).

Our main goal in this article is to prove the convergence of the numerical solution obtained by the compact scheme R3C to the unique viscosity solution of (1)-(5). In the literature, unlike for many standard finite difference schemes, there are very few results concerning the convergence of high-order compact finite difference schemes. In [7] compact finite difference methods for initial-boundary-value problems for mixed systems of strongly parabolic and strictly hyperbolic equations are studied. Assuming the existence of a smooth solution, a pilot function $[25,30]$ is constructed which leads to convergence results. Il'in [18] studies compact finite difference schemes for linear convection-diffusion equations and gives error estimates. Wang and Liu [31] propose a fourthorder scheme for the two-dimensional, incompressible Navier-Stokes equations in vorticity formulation and prove its convergence using energy estimates. The convergence of approximation schemes for fully nonlinear second order equations is studied in a general setting in [4]. The originality of this paper consists in the combination of high-order compact finite difference schemes and techniques for viscosity solutions.

This article is organized in the following way. To provide the tools needed later, we study in Section 2 the properties of the discretization matrices and prove the positivity of numerical solutions. Since the numerical solution involves an approximation process for (3), we prove in Section 3.1 an analytical convergence result using the "half-relaxed limits" technique. Finally, in Section 3.2, we show our main result, the convergence of the compact scheme R3C, using the results of [4]. 


\section{Compact scheme R3C}

In this section we reformulate the problem (1)-(5) using a variable transformation, recall the compact finite difference scheme R3C [13] and provide the tools for the convergence results in the next section.

\subsection{Definition of the scheme}

To overcome a possible degeneration at $S=0$ and to obtain a forward parabolic problem, we use the variable transformations

Equation (1) is hereby transformed into

$$
x(S)=\ln \left(\frac{S}{E}\right), \quad t(\tau)=\frac{1}{2} \sigma_{0}^{2}\left(\tau_{0}-\tau\right), \quad u=\exp (-x) \frac{V}{E} .
$$

$$
u_{t}-\left(1+\Psi\left[\exp (K t+x) a^{2} E\left(u_{x x}+u_{x}\right)\right]\right)\left(u_{x x}+u_{x}\right)-K u_{x}=0,
$$

with

$$
x \in \mathbb{R}, \quad 0 \leq t \leq T=\sigma_{0}^{2} \tau_{0} / 2, \quad K=\frac{2 \rho}{\sigma_{0}^{2}}
$$

For the computation we replace $\mathbb{R}$ by $\bar{\Omega}=[-R, R]$ with $R>0$. For simplicity, we consider a uniform grid $Z=\left\{x_{i} \in[-R, R]: x_{i}=i h, i=-N, \ldots, N\right\}$ consisting of $2 N+1$ grid points, with $R=N h$ and with space step $h$ and time step $k$, where $T=M k$. Let $U_{i}^{n}$ denote the approximate solution of (6) in $x_{i}$ at time $t_{n}=n k$ and set $U^{n}=\left(U_{i}^{n}\right)_{i=1}^{2 N+1}$ and $U=\left(U^{n}\right)_{n=1}^{M}$.

The problem is completed by the following initial and boundary conditions

$$
\begin{aligned}
u(x, 0) & =\max (1-\exp (-x), 0), \\
u(-R, t) & =0, \\
u(R, t) & =1-\exp (-R-K t) .
\end{aligned}
$$

The latter condition corresponds to the asymptotic value of the exact solution of the equation for $a=0$. More precisely, the solution of (6) satisfies (see (5))

$$
u(x, t) \sim 1-\exp (-x-K t) \quad \text { as } x \rightarrow \infty .
$$

Approximately, we expect to have $u(R, t) \approx 1-\exp \left(-x_{N}-K t\right)$ for sufficiently large $R>0$. The nonlinear correction of the volatility in (1) is a function of the second derivative, so we assume that the influence of the nonlinearity at the boundary can be neglected for large $R$. The error caused by boundary conditions imposed on an artificial boundary for a class of Black-Scholes equations has been studied rigorously in [21].

We use a Dormand-Prince-4-5 Runge-Kutta scheme to solve the ordinary differential equation (3) and a cubic spline interpolation to obtain the values of $\Psi$ for arbitrary arguments.

With

$$
\beta=1+\Psi\left[\exp \left(K t+x_{i}\right) a^{2} E\left(\Delta_{2} U_{i}^{n}+\Delta_{0} U_{i}^{n}\right)\right], \quad \lambda=\beta+K,
$$

where

$$
\Delta_{0} U_{i}^{n}=\frac{U_{i+1}^{n}-U_{i-1}^{n}}{2 h}, \quad \Delta_{2} U_{i}^{n}=\frac{U_{i+1}^{n}-2 U_{i}^{n}+U_{i-1}^{n}}{h^{2}},
$$

the "semi-discretized" equation (6) at $x=x_{i}$ takes the form $u_{t}=\beta u_{x x}-\lambda u_{x}$. Below we study this equation for arbitrary values $\beta, \lambda>0$. We use the following abbreviations

$$
\alpha=\frac{\lambda h}{2}, \quad r=\frac{k}{h^{2}}, \quad \mu=\frac{\lambda k}{h} .
$$


We define the two-level three-point scheme R3C as in [13] by

$$
D_{t} U_{i}^{n}=\beta\left(\frac{1}{2}+A_{1}\right) \Delta_{2} U_{i}^{n}+\beta\left(\frac{1}{2}+A_{2}\right) \Delta_{2} U_{i}^{n+1}-\lambda\left(\frac{1}{2}+B_{1}\right) \Delta_{0} U_{i}^{n}-\lambda\left(\frac{1}{2}+B_{2}\right) \Delta_{0} U_{i}^{n+1},
$$

where $D_{t} U_{i}^{n}=\left(U_{i}^{n+1}-U_{i}^{n}\right) / k$, and $A_{i}, B_{i}$ are real constants given by

$$
\begin{aligned}
& B_{1}=-B_{2}, \\
& A_{1}=-\frac{1}{12 k \beta}\left(-2 h^{2}+6 \lambda^{2} k^{2} B_{2}-k^{2} \lambda^{2}-12 k \beta B_{2}\right), \\
& A_{2}=-\frac{1}{12 k \beta}\left(2 h^{2}+6 \lambda^{2} k^{2} B_{2}+k^{2} \lambda^{2}+12 k \beta B_{2}\right), \\
& B_{2}=-\frac{1+4 r^{2} \alpha^{2}}{12 \beta r}
\end{aligned}
$$

Then the R3C scheme can be written in the form

$$
A^{n} U^{n+1}=B^{n} U^{n}, \quad A^{n}=\left[a_{-1}, a_{0}, a_{1}\right], \quad B^{n}=\left[b_{-1}, b_{0}, b_{1}\right],
$$

where the notation $[a, b, c]$ denotes a tridiagonal matrix whose diagonals have constant entries $a, b$, and $c$, respectively. The coefficients $a_{i}, b_{i}$ are given by

$$
\begin{aligned}
a_{-1} & =-\beta\left(\frac{r}{2}+r A_{2}\right)-\frac{\mu}{4}-\mu \frac{B_{2}}{2}, & b_{-1} & =\beta\left(\frac{r}{2}+r A_{1}\right)+\frac{\mu}{4}+\mu \frac{B_{1}}{2}, \\
a_{0} & =1+\beta\left(r+2 r A_{2}\right), & b_{0} & =1-\beta\left(r+2 r A_{1}\right), \\
a_{1} & =-\beta\left(\frac{r}{2}+r A_{2}\right)+\frac{\mu}{4}+\mu \frac{B_{2}}{2}, & b_{1} & =\beta\left(\frac{r}{2}+r A_{1}\right)-\frac{\mu}{4}-\mu \frac{B_{1}}{2},
\end{aligned}
$$

or, more explicitly, by

$$
\begin{aligned}
a_{-1} & =-\frac{12 r \beta^{2}-2 \beta+r \lambda^{2} h^{2}+r^{3} \lambda^{4} h^{4}+6 r \lambda h \beta-\lambda h-r^{2} \lambda^{3} h^{3}}{24 \beta}, \\
a_{0} & =\frac{10 \beta+12 r \beta^{2}+r \lambda^{2} h^{2}+r^{3} \lambda^{4} h^{4}}{12 \beta}, \\
a_{1} & =-\frac{12 r \beta^{2}-2 \beta+r \lambda^{2} h^{2}+r^{3} \lambda^{4} h^{4}-6 r \lambda h \beta+\lambda h+r^{2} \lambda^{3} h^{3}}{24 \beta}, \\
b_{-1} & =\frac{12 r \beta^{2}+2 \beta+r \lambda^{2} h^{2}+r^{3} \lambda^{4} h^{4}+6 r \lambda h \beta+\lambda h+r^{2} \lambda^{3} h^{3}}{24 \beta} \\
b_{0} & =-\frac{-10 \beta+12 r \beta^{2}+r \lambda^{2} h^{2}+r^{3} \lambda^{4} h^{4}}{12 \beta} \\
b_{1} & =\frac{12 r \beta^{2}+2 \beta+r \lambda^{2} h^{2}+r^{3} \lambda^{4} h^{4}-6 r \lambda h \beta-\lambda h-r^{2} \lambda^{3} h^{3}}{24 \beta} .
\end{aligned}
$$

\subsection{Properties of the scheme}

We recall the following result from [13].

Theorem 1 [13]. The resulting scheme $R 3 C$ is an unconditionally stable (in the sense of von Neumann), non-oscillatory and forward diffusive scheme of order $\mathcal{O}\left(k^{2}+h^{4}\right)$. 
In the following we prove some properties of the discretization matrices needed in the convergence proof in Section 3. To simplify the presentation, we only consider the case $K=0$ which corresponds to zero interest rate. Notice that this implies $\lambda=\beta$. In the general case, similar conditions as in Lemma 2 below can be obtained, with bounds depending on $h, \beta$, and $\lambda$.

Lemma 2. If $h<2$ and

$$
\frac{1}{6 \beta} \leq r<\frac{1}{2 \beta}
$$

then $B^{n}$ is a positive matrix (i.e., all elements are positive) and $A^{n}$ is an M-matrix. More specifically, $a_{0}, b_{-1}, b_{0}, b_{1}$ are positive, $a_{-1}, a_{1}$ are negative or zero, $A^{n}$ is non-singular, and $\left(A^{n}\right)^{-1}$ is a positive matrix.

Proof. The coefficients $a_{0}, b_{-1}$ are always positive. It follows from (14) that $b_{0}$ and $b_{1}$ are positive if

$$
\begin{array}{r}
10 \beta-12 r \beta^{2}-r \beta^{2} h^{2}-\beta^{4} h^{4} r^{3}>0, \\
12 r \beta+2+r \beta h^{2}+\beta^{3} h^{4} r^{3}-6 \beta h r-h-\beta^{2} h^{3} r^{2}>0,
\end{array}
$$

respectively, and $a_{-1}, a_{1}$ are negative or zero if

$$
\begin{aligned}
& 12 r \beta^{2}-2 \beta+r \beta^{2} h^{2}+\beta^{4} h^{4} r^{3}+6 \beta^{2} h r-\beta h-\beta^{3} h^{3} r^{2} \geq 0, \\
& 12 r \beta^{2}-2 \beta+r \beta^{2} h^{2}+\beta^{4} h^{4} r^{3}-6 \beta^{2} h r+\beta h+\beta^{3} h^{3} r^{2} \geq 0,
\end{aligned}
$$

respectively.

First, we study (17). Consider the polynomial $p(\beta)=h^{4} r^{3} \beta^{2}-h^{3} r^{2} \beta+r h^{2}-6 h r+12 r$. It is positive for all $h \neq 4$, since its leading coefficient is positive and its discriminant is $-3 r^{4} h^{4}(h-4)^{2}$, which is negative for $h \neq 4$. Hence, $p(\beta) \beta+2-h>0$ and thus (17) follows if $h<2$.

We solve the equations related to (16), (18), (19) for $r$, being cubic polynomials in $r$. For each equation we obtain one real root and two complex roots. From the real root of the first equation we obtain the condition $r<c_{0}(h) / \beta$ with

$$
c_{0}(h)=\frac{1}{3} \frac{x^{2 / 3}-36-3 h^{2}}{h^{2} x^{1 / 3}}
$$

where $x=135 h^{2}+3 \sqrt{3} \sqrt{1728+432 h^{2}+711 h^{4}+h^{6}}$. The function $c_{0}$ is decreasing in $h$ with $\min _{h \in[0,2]} c_{0}(h)=$ $\frac{1}{2}$, which gives the upper bound in (15).

The real roots of the other equations result in the condition $\max \left(c_{1}(h), c_{2}(h)\right) / \beta \leq r$ with

$$
\begin{aligned}
& c_{1}(h)=\frac{1}{3} \frac{y^{1 / 3}}{h^{2}}-\frac{2}{3} \frac{18+h^{2}+9 h}{h^{2} y^{1 / 3}}+\frac{1}{3 h}, \\
& c_{2}(h)=\frac{1}{3} \frac{z^{1 / 3}}{h^{2}}-\frac{2}{3} \frac{18+h^{2}-9 h}{h^{2} z^{1 / 3}}-\frac{1}{3 h},
\end{aligned}
$$

where

$$
\begin{aligned}
& y=-54 h+10 h^{3}+6 \sqrt{3} \sqrt{432+423 h^{2}+648 h+12 h^{4}+126 h^{3}+h^{6}+2 h^{5}}, \\
& z=54 h-10 h^{3}+6 \sqrt{3} \sqrt{432+423 h^{2}-648 h+12 h^{4}-126 h^{3}+h^{6}-2 h^{5}}
\end{aligned}
$$

It can be seen that the functions $c_{1}$ and $c_{2}$ both attain their maximum at $h=0$ with $c_{1}(0)=c_{2}(0)=\frac{1}{6}$. This yields the lower bound in (15). Therefore, $B^{n}$ is a positive matrix and $A^{n}$ is an L-matrix if (15) holds. Since $a_{0}>\left|a_{-1}\right|+\left|a_{1}\right|, A^{n}$ is strictly diagonally dominant. Hence, $A^{n}$ is an M-matrix which yields the claim. 


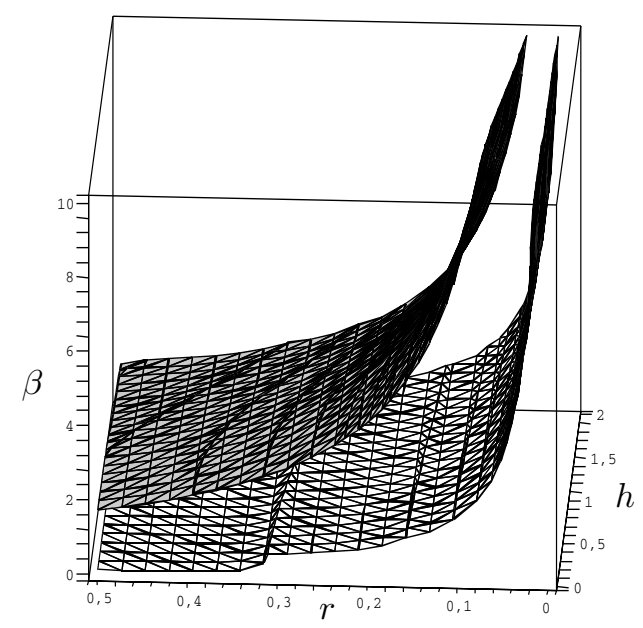

FiguRE 1. The two surfaces represent the equations $r=c_{0}(h) / \beta$ and $r=\max \left(c_{1}(h), c_{2}(h)\right) / \beta$.

In Figure 1 the set $\max \left(c_{1}(h), c_{2}(h)\right) / \beta \leq r \leq c_{0}(h) / \beta$ is shown. As a by-product of Lemma 2 , we obtain the following corollary, which ensures the positivity of the numerical solutions.

Corollary 3. Let the assumptions of Lemma 2 hold. Then the linear, constant coefficient R3C scheme is positive, i.e. for all $n \in \mathbb{N}$ :

$$
U^{n} \geq 0 \Longrightarrow U^{n+1} \geq 0,
$$

where the inequality holds for all components of the vectors.

Remark 4. A finite difference scheme of the form (12) is called positive if $\left(A^{n}\right)^{-1} B^{n}$ is a positive matrix. Unlike for many second-order schemes, the matrices $A^{n}$ and $B^{n}$ resulting from fourth-order schemes generally do not commute and positivity cannot be easily deduced. The positivity of the scheme holds if both matrices $\left(A^{n}\right)^{-1}$ and $B^{n}$ are positive.

Remark 5. The conditions of Lemma 2 are sufficient but not necessary. Frequently, such conditions are too restrictive in practice and the scheme will preserve the positivity for a larger set of discretization parameters [27]. We observed this also in our numerical experiments presented in [13].

\section{Convergence Results}

For the convenience of the reader, we briefly recall the notion of viscosity solutions, introduced by Crandall and Lions [10]. For a general presentation on viscosity solutions we refer to [11]. Following the notation of [4], we can write $(6)$ as

$$
G\left(x, t, u(x, t), u_{t}(x, t), u_{x}(x, t), u_{x x}(x, t)\right)=0 \quad \text { in } \bar{\Omega} \times[0, T],
$$

where $G$ is given by

$$
\begin{aligned}
& G\left(x, t, u(x, t), u_{t}(x, t), u_{x}(x, t), u_{x x}(x, t)\right)= \\
& \begin{cases}u_{t}-\left(1+\Psi\left[\exp (K t+x) a^{2} E\left(u_{x x}+u_{x}\right)\right]\right)\left(u_{x x}+u_{x}\right)-K u_{x} & \text { in } Q_{t}, \\
u(x, 0)-\max (1-\exp (-x), 0) & \text { in } \Omega, \\
u(-R, t) & \text { in }(0, T), \\
u(R, t)-(1-\exp (-R-K t)) & \text { in }(0, T),\end{cases}
\end{aligned}
$$


where $Q_{t}=\Omega \times(0, T)$. Although we have assumed $K=0$ in the previous section, the results of this section hold for any $K \geq 0$ provided that the conclusion of Lemma 2 holds. In the following, let $z^{*}$ and $z_{*}$ denote the upper semi-continuous and lower semi-continuous envelope of the function $z: C \rightarrow \mathbb{R}$, where $C$ is a closed subset of $\mathbb{R}$, defined by

$$
z^{*}(x)=\limsup _{y \rightarrow x, y \in C} z(y), \quad z_{*}(x)=\liminf _{y \rightarrow x, y \in C} z(y) .
$$

Definition 6. A locally bounded function $u: \bar{\Omega} \rightarrow \mathbb{R}$ is a viscosity subsolution (respectively supersolution) of (20) if and only if for all $\varphi \in C^{2}(\bar{\Omega} \times[0, T])$ and for all maximum (respectively minimum) points $(x, t)$ of $u^{*}-\varphi$ (respectively $u_{*}-\varphi$ ), one has

$$
\begin{aligned}
G_{*}\left(x, t, u^{*}(x, t), \varphi_{t}(x, t), \varphi_{x}(x, t), \varphi_{x x}(x, t)\right) & \leq 0 \\
\left(\text { respectively } G^{*}\left(x, t, u_{*}(x, t), \varphi_{t}(x, t), \varphi_{x}(x, t), \varphi_{x x}(x, t)\right)\right. & \geq 0 .)
\end{aligned}
$$

A locally bounded function is a viscosity solution of (20) if it is a viscosity subsolution and a viscosity supersolution.

\subsection{Analytical convergence result}

The solution of (6) involves two approximation processes. One is imposing the Dirichlet boundary conditions (8) and (9). The existence and uniqueness proof in [3] uses the boundary conditions (5). It is easy to carry over the existence and uniqueness proof with only small changes, so we omit the proof. The convergence of the solution on a bounded domain to the solution on the half-space has been studied in $[5,22]$ for the linear case, i.e. $a=0$.

The other approximation arises when solving (3). Since the right-hand side of (3) is unbounded for $A \rightarrow 0$, it is necessary to solve the ordinary differential equation approximately with a bounded approximation of the right-hand side. This gives rise to an approximate function $\Psi_{\varepsilon}$ which is used in the numerical solution of (6). Thus, we are in fact solving an approximate problem,

$$
u_{t}-\left(1+\Psi_{\varepsilon}\left[\exp (K t+x) a^{2} E\left(u_{x x}+u_{x}\right)\right]\right)\left(u_{x x}+u_{x}\right)-K u_{x}=0,
$$

with (7)-(9). In the following we show that the solution of this approximate problem converges to the solution of the original problem.

Proposition 7. Let $\Psi_{\varepsilon}$ be a monotone smooth approximation of $\Psi$ with bounded derivative such that $\Psi_{\varepsilon} \rightarrow \Psi$ locally uniformly as $\varepsilon \rightarrow 0$. Then the viscosity solution $u_{\varepsilon}$ of (21) and (7)-(9) converges to the viscosity solution $u$ of (6) and (7)-(9) as $\varepsilon \rightarrow 0$.

Proof. We use the "half-relaxed limits" technique which has been introduced by Barles and Perthame $[1,2]$ and Ishii [19]. Let $u_{\varepsilon}$ denote a solution to the approximate problem (21), (7)-(9) with $\varepsilon>0$. We omit an existence proof which is very similar to the one for the original problem. Since $u_{1} \equiv 1$ and $u_{2} \equiv 0$ are superand subsolutions, respectively, comparison arguments show that $u_{\varepsilon}$ is bounded independently of $\varepsilon$. Then

$$
\begin{aligned}
& \bar{u}(x, t)=\limsup _{\varepsilon \rightarrow 0} u_{\varepsilon}(x, t)=\limsup _{\varepsilon^{\prime} \rightarrow 0}\left\{u_{\varepsilon}\left(x^{\prime}, t^{\prime}\right): \varepsilon \leq \varepsilon^{\prime},\left\|(x, t)-\left(x^{\prime}, t^{\prime}\right)\right\| \leq \varepsilon^{\prime}\right\}, \\
& \underline{u}(x, t)=\liminf _{\varepsilon \rightarrow 0} u_{\varepsilon}(x, t)=\liminf _{\varepsilon^{\prime} \rightarrow 0}\left\{u_{\varepsilon}\left(x^{\prime}, t^{\prime}\right): \varepsilon \leq \varepsilon^{\prime},\left\|(x, t)-\left(x^{\prime}, t^{\prime}\right)\right\| \leq \varepsilon^{\prime}\right\},
\end{aligned}
$$

are well-defined. By ([11], Lemma 6.1) both limits are discontinuous viscosity solutions of (6), (7)-(9), since $\Psi_{\varepsilon} \rightarrow \Psi$ locally uniformly as $\varepsilon \rightarrow 0$. The strong comparison result for (6) in ([3], App. B, pp. 395) shows that $\bar{u}=\underline{u}=u$. 


\subsection{Convergence of the compact scheme}

The convergence of approximation schemes for fully nonlinear parabolic equations has been studied in an abstract setting in [4]. We want to apply Theorem 2.1 in [4] to show the convergence of the compact scheme R3C to the viscosity solution of (21), (7)-(9). We start by recalling the assumptions of Theorem 2.1 in [4]. The numerical scheme R3C (11) approximating (20) can be written as

$$
S\left(k, h, n, i, U_{i}^{n+1}, U\right)=0,
$$

where $U_{i}^{n+1}$ is the desired approximate solution that is computed using elements of $U$. Roughly speaking, Theorem 2.1 in [4] states that any stable, consistent and monotone scheme converges to the solution of (20), provided (20) satisfies a "strong uniqueness" condition. Therefore the scheme $S$ is expected to have the following properties, at least for some sequence $(k, h)$ converging to zero.

(S1) For all $(k, h)$, there exists a solution $U$ of $(22)$ that is bounded independently of $(k, h)$.

(S2) For any smooth function $\phi$ and for any $(x, t)$ in $\bar{\Omega} \times[0, T]$, it holds

$$
\begin{aligned}
&\left.\liminf _{(k, h) \rightarrow 0,} x_{i}, t_{n}\right) \rightarrow(x, t), \xi \rightarrow 0 \frac{S\left(k, h, n, i, \phi_{i}^{n+1}+\xi, \phi+\xi\right)}{\rho(k, h)} \geq G_{*}\left(x, t, \phi(x, t), \phi_{t}(x, t), \phi_{x}(x, t), \phi_{x x}(x, t)\right), \\
& \limsup _{(k, h) \rightarrow 0,\left(x_{i}, t_{n}\right) \rightarrow(x, t), \xi \rightarrow 0} \frac{S\left(k, h, n, i, \phi_{i}^{n+1}+\xi, \phi+\xi\right)}{\rho(k, h)} \leq G^{*}\left(x, t, \phi(x, t), \phi_{t}(x, t), \phi_{x}(x, t), \phi_{x x}(x, t)\right),
\end{aligned}
$$

for some function $\rho(k, h)>0$ such that $\rho(k, h) \rightarrow 0$ as $(k, h) \rightarrow 0$.

(S3) If $U \geq V$ (the inequality holds for all components) and $U_{i}^{n+1}=V_{i}^{n+1}$, then

$$
S\left(k, h, n, i, U_{i}^{n+1}, U\right) \leq S\left(k, h, n, i, V_{i}^{n+1}, V\right)
$$

for any $k, h>0,1 \leq n \leq M, 1 \leq i \leq 2 N+1$ and for all $U, V \in \mathbb{R}^{M(2 N+1)}$.

(S4) If the locally bounded upper semi-continuous (lower semi-continuous) function $u(v)$ is a viscosity subsolution (supersolution) of (20) then

$$
u \leq v \text { in } \bar{\Omega} \text {. }
$$

Our main result on the convergence of the compact scheme $\mathrm{R} 3 \mathrm{C}$ is the following theorem.

Theorem 8. Assume that $\Psi^{\prime}$ is bounded, the constant transaction cost parameter a is sufficiently small (see below) and the assumptions of Lemma 2 are fulfilled. Then the solution $U$ converges to the unique viscosity solution of $(21),(7)-(9)$ as $(k, h) \rightarrow 0$, uniformly on each compact subset of $\bar{\Omega}$.

Proof. In order to be able to apply Theorem 2.1 in [4], we have to check the assumptions (S1)-(S4). The proof of (S4) is given in ([3], App. B, pp. 395).

We show that $\left\|U^{n}\right\|_{\infty}$ is bounded for arbitrary $n \in \mathbb{N}$ if $\left\|U^{0}\right\|_{\infty}$ is bounded. For arbitrary $n \in \mathbb{N}$ let $i_{0} \in$ $\{-N, \ldots, N\}$ be such that $\left\|U^{n+1}\right\|_{\infty}=\left|U_{i_{0}}^{n+1}\right|$. Employing Lemma 2 and using $a_{-1}+a_{0}+a_{1}=b_{-1}+b_{0}+b_{1}=1$, we can estimate

$$
\begin{aligned}
\left\|U^{n+1}\right\|_{\infty} & =\left|U_{i_{0}}^{n+1}\right|=a_{-1}\left|U_{i_{0}}^{n+1}\right|+a_{0}\left|U_{i_{0}}^{n+1}\right|+a_{1}\left|U_{i_{0}}^{n+1}\right| \\
& \leq a_{-1}\left|U_{i_{0}-1}^{n+1}\right|+a_{0}\left|U_{i_{0}}^{n+1}\right|+a_{1}\left|U_{i_{0}+1}^{n+1}\right| \\
& \leq\left|a_{-1} U_{i_{0}-1}^{n+1}+a_{0} U_{i_{0}}^{n+1}+a_{1} U_{i_{0}+1}^{n+1}\right| \\
& =\left|b_{-1} U_{i_{0}-1}^{n}+b_{0} U_{i_{0}}^{n}+b_{1} U_{i_{0}+1}^{n}\right| \\
& \leq\left\|B^{n} U^{n}\right\|_{\infty} \leq\left\|B^{n}\right\|_{\infty}\left\|U^{n}\right\|_{\infty}=\left\|U^{n}\right\|_{\infty},
\end{aligned}
$$

where $\left\|B^{n}\right\|_{\infty}$ is the row-sum norm of $B^{n}$. Thus, $\left\|U^{n}\right\|_{\infty} \leq\left\|U^{0}\right\|_{\infty}$ for $n \in \mathbb{N}$, yielding (S1). 
The consistency assumption (S2) follows from Theorem 1. It remains to show that (S3) holds. For simplicity, we will only consider the case $K=0$. This relates to the case of zero interest rate in the financial model. The case $K>0$ can be proved analogously. Define $F: \mathbb{R}^{4} \rightarrow \mathbb{R}$ by

$$
\begin{aligned}
F\left(\Delta_{2} U_{i}^{n+1}, \Delta_{0} U_{i}^{n+1}, \Delta_{2} U_{i}^{n}, \Delta_{0} U_{i}^{n}\right) & = \\
\beta & {\left[\left(\frac{1}{2}+A_{1}\right) \Delta_{2} U_{i}^{n}+\left(\frac{1}{2}+A_{2}\right) \Delta_{2} U_{i}^{n+1}-\left(\frac{1}{2}+B_{1}\right) \Delta_{0} U_{i}^{n}-\left(\frac{1}{2}+B_{2}\right) \Delta_{0} U_{i}^{n+1}\right] . }
\end{aligned}
$$

Note that $\beta, A_{1}, A_{2}, B_{1}, B_{2}$ depend on $U$ as well. Using this definition we can write (11) as

$$
S\left(k, h, n, i, U_{i}^{n+1}, U\right)=U_{i}^{n+1}-U_{i}^{n}-k F\left(\Delta_{2} U_{i}^{n+1}, \Delta_{0} U_{i}^{n+1}, \Delta_{2} U_{i}^{n}, \Delta_{0} U_{i}^{n}\right) .
$$

Let $U, V \in \mathbb{R}^{M(2 N+1)}$ with $U \geq V$ and $U_{i}^{n+1}=V_{i}^{n+1}$. We need to show that

$$
S\left(k, h, n, i, V_{i}^{n+1}, V\right)-S\left(k, h, n, i, U_{i}^{n+1}, U\right) \geq 0 .
$$

With the abbreviations $W_{i}^{n+1}=U_{i}^{n+1}-V_{i}^{n+1}, W_{i}^{n}=U_{i}^{n}-V_{i}^{n}$ we infer

$$
\begin{aligned}
S\left(k, h, n, i, V_{i}^{n+1}, V\right)-S\left(k, h, n, i, U_{i}^{n+1}, U\right)= & \left(U_{i}^{n}-V_{i}^{n}\right)+k\left[F\left(\Delta_{2} U_{i}^{n+1}, \Delta_{0} U_{i}^{n+1}, \Delta_{2} U_{i}^{n}, \Delta_{0} U_{i}^{n}\right)\right. \\
& \left.-F\left(\Delta_{2} V_{i}^{n+1}, \Delta_{0} V_{i}^{n+1}, \Delta_{2} V_{i}^{n}, \Delta_{0} V_{i}^{n}\right)\right] \\
= & W_{i}^{n}+k \nabla F(z)\left(\Delta_{2} W_{i}^{n+1}, \Delta_{0} W_{i}^{n+1}, \Delta_{2} W_{i}^{n}, \Delta_{0} W_{i}^{n}\right),
\end{aligned}
$$

using the mean value theorem

$$
\begin{aligned}
F\left(\Delta_{2} U_{i}^{n+1}, \Delta_{0} U_{i}^{n+1}, \Delta_{2} U_{i}^{n}, \Delta_{0} U_{i}^{n}\right)-F\left(\Delta_{2} V_{i}^{n+1}, \Delta_{0} V_{i}^{n+1}, \Delta_{2} V_{i}^{n}, \Delta_{0} V_{i}^{n}\right) \\
=\nabla F(z)\left[\left(\Delta_{2} U_{i}^{n+1}, \Delta_{0} U_{i}^{n+1}, \Delta_{2} U_{i}^{n}, \Delta_{0} U_{i}^{n}\right)-\left(\Delta_{2} V_{i}^{n+1}, \Delta_{0} V_{i}^{n+1}, \Delta_{2} V_{i}^{n}, \Delta_{0} V_{i}^{n}\right)\right]
\end{aligned}
$$

for some

$$
z \in\left[\left(\Delta_{2} U_{i}^{n+1}, \Delta_{0} U_{i}^{n+1}, \Delta_{2} U_{i}^{n}, \Delta_{0} U_{i}^{n}\right),\left(\Delta_{2} V_{i}^{n+1}, \Delta_{0} V_{i}^{n+1}, \Delta_{2} V_{i}^{n}, \Delta_{0} V_{i}^{n}\right)\right],
$$

where $z=\left(z_{1}, z_{2}, z_{3}, z_{4}\right)$, and $[p, q]$ denotes the line between $p, q \in \mathbb{R}^{4}$. We compute

$$
\nabla F(z)=\left(\begin{array}{c}
\beta\left(\frac{1}{2}+A_{2}\right) \\
-\beta\left(\frac{1}{2}+B_{2}\right) \\
\beta\left[\left(\frac{1}{2}+A_{1}\right)+c\left(a^{2}\right) A_{1, \beta} \Psi^{\prime} z_{3}\right]+c\left(a^{2}\right) \Psi^{\prime}\left(\frac{1}{2}+A_{1}\right) z_{3} \\
-\beta\left[\left(\frac{1}{2}+B_{1}\right)+c\left(a^{2}\right) B_{1, \beta} \Psi^{\prime} z_{4}\right]-c\left(a^{2}\right) \Psi^{\prime}\left(\frac{1}{2}+B_{1}\right) z_{4}
\end{array}\right)
$$

where $\beta, A_{1}, A_{2}, B_{1}, B_{2}, \Psi^{\prime}$ and $A_{1, \beta}, B_{1, \beta}$, the derivatives with respect to $\beta$, are evaluated in $z$ and $c\left(a^{2}\right)$ is a positive constant depending on $a^{2}$ with $c\left(a^{2}\right) \rightarrow 0$ as $a \rightarrow 0$. We obtain

$$
S\left(k, h, n, i, V_{i}^{n+1}, V\right)-S\left(k, h, n, i, U_{i}^{n+1}, U\right)=W_{i}^{n}+k \beta T_{1}+c\left(a^{2}\right) k T_{2},
$$

where

$$
\begin{aligned}
& T_{1}=\left(\frac{1}{2}+A_{2}\right) \frac{W_{i+1}^{n+1}-2 W_{i}^{n+1}+W_{i-1}^{n+1}}{h^{2}}-\left(\frac{1}{2}+B_{2}\right) \frac{W_{i+1}^{n+1}-W_{i-1}^{n+1}}{2 h}+\left(\frac{1}{2}+A_{1}\right) \frac{W_{i+1}^{n}-2 W_{i}^{n}+W_{i-1}^{n}}{h^{2}} \\
& -\left(\frac{1}{2}+B_{1}\right) \frac{W_{i+1}^{n}-W_{i-1}^{n}}{2 h}
\end{aligned}
$$


and

$$
T_{2}=\left[\beta A_{1, \beta} \Psi^{\prime}+\Psi^{\prime}\left(\frac{1}{2}+A_{1}\right)\right] z_{3} \frac{W_{i+1}^{n}-2 W_{i}^{n}+W_{i-1}^{n}}{h^{2}}-\left[\beta B_{1, \beta} \Psi^{\prime}+\Psi^{\prime}\left(\frac{1}{2}+B_{1}\right)\right] z_{4} \frac{W_{i+1}^{n}-W_{i-1}^{n}}{2 h} .
$$

The term $T_{1}$ collects the terms known from the linear scheme (13), where $\beta$ is simply a positive constant. The term $T_{2}$ involves additional expressions for the nonlinear case. Note also that the nonlinear terms only involve the coefficients at time level $n$, since the nonlinearity is discretized explicitly. We will make use of this observation in the following by employing Lemma 2 to obtain the positivity of $T_{1}$ and use this to control the term $T_{2}$ for suitably small values of $a$.

We collect the terms in (23) according to the grid points, make use of $W_{i}^{n+1}=0$ and obtain

$$
\begin{aligned}
S(k, & \left.h, n, i, V_{i}^{n+1}, V\right)-S\left(k, h, n, i, U_{i}^{n+1}, U\right)=\left[1-r \beta\left(1+2 A_{1}\right)+c\left(a^{2}\right) 2 r\left(\beta A_{1, \beta} \Psi^{\prime}+\Psi^{\prime}\left(\frac{1}{2}+A_{1}\right)\right) z_{3}\right] W_{i}^{n} \\
& +\left[r \beta\left(\frac{1}{2}+A_{2}\right)-\frac{\mu}{2}\left(\frac{1}{2}+B_{2}\right)\right] W_{i+1}^{n+1}+\left[r \beta\left(\frac{1}{2}+A_{2}\right)+\frac{\mu}{2}\left(\frac{1}{2}+B_{2}\right)\right] W_{i-1}^{n+1}+\left[r \beta\left(\frac{1}{2}+A_{1}\right)\right. \\
& \left.-\frac{\mu}{2}\left(\frac{1}{2}+B_{1}\right)\right] W_{i+1}^{n}+c\left(a^{2}\right)\left[r\left(\beta A_{1, \beta} \Psi^{\prime}+\Psi^{\prime}\left(\frac{1}{2}+A_{1}\right)\right) z_{3}-\frac{k}{2 h}\left(\beta B_{1, \beta} \Psi^{\prime}+\Psi^{\prime}\left(\frac{1}{2}+B_{1}\right)\right) z_{4}\right] W_{i+1}^{n} \\
& +\left[r \beta\left(\frac{1}{2}+A_{1}\right)+\frac{\mu}{2}\left(\frac{1}{2}+B_{1}\right)\right] W_{i-1}^{n}+c\left(a^{2}\right)\left[r\left(\beta B_{1, \beta} \Psi^{\prime}+\Psi^{\prime}\left(\frac{1}{2}+A_{1}\right)\right) z_{3}\right. \\
& \left.+\frac{k}{2 h}\left(\beta B_{1, \beta} \Psi^{\prime}+\Psi^{\prime}\left(\frac{1}{2}+B_{1}\right)\right) z_{4}\right] W_{i-1}^{n} \\
= & {\left[b_{0}+c\left(a^{2}\right) 2 r\left(\beta A_{1, \beta} \Psi^{\prime}+\Psi^{\prime}\left(\frac{1}{2}+A_{1}\right)\right) z_{3}\right] W_{i}^{n}-a_{i+1} W_{i+1}^{n+1}-a_{i-1} W_{i-1}^{n+1} } \\
& +\left[b_{1}+c\left(a^{2}\right)\left[r\left(\beta A_{1, \beta} \Psi^{\prime}+\Psi^{\prime}\left(\frac{1}{2}+A_{1}\right)\right) z_{3}-\frac{k}{2 h}\left(\beta B_{1, \beta} \Psi^{\prime}+\Psi^{\prime}\left(\frac{1}{2}+B_{1}\right)\right) z_{4}\right]\right] W_{i+1}^{n} \\
& +\left[b_{-1}+c\left(a^{2}\right)\left[r\left(\beta A_{1, \beta} \Psi^{\prime}+\Psi^{\prime}\left(\frac{1}{2}+A_{1}\right)\right) z_{3}+\frac{k}{2 h}\left(\beta B_{1, \beta} \Psi^{\prime}+\Psi^{\prime}\left(\frac{1}{2}+B_{1}\right)\right) z_{4}\right]\right] W_{i-1}^{n} \\
\geq & {\left[b_{0}+c\left(a^{2}\right) 2 r\left(\beta A_{1, \beta} \Psi^{\prime}+\Psi^{\prime}\left(\frac{1}{2}+A_{1}\right)\right) z_{3}\right] W_{i}^{n}+\left[b_{1}+c\left(a^{2}\right)\left[r\left(\beta A_{1, \beta} \Psi^{\prime}+\Psi^{\prime}\left(\frac{1}{2}+A_{1}\right)\right) z_{3}\right.\right.} \\
& \left.\left.-\frac{k}{2 h}\left(\beta B_{1, \beta} \Psi^{\prime}+\Psi^{\prime}\left(\frac{1}{2}+B_{1}\right)\right) z_{4}\right]\right] W_{i+1}^{n}+\left[b_{-1}+c\left(a^{2}\right)\left[r\left(\beta A_{1, \beta} \Psi^{\prime}+\Psi^{\prime}\left(\frac{1}{2}+A_{1}\right)\right) z_{3}\right.\right. \\
& \left.\left.+\frac{k}{2 h}\left(\beta B_{1, \beta} \Psi^{\prime}+\Psi^{\prime}\left(\frac{1}{2}+B_{1}\right)\right) z_{4}\right]\right] W_{i-1}^{n}
\end{aligned}
$$

where $a_{-1}, a_{1}, b_{-1}, b_{0}, b_{1}$ are the coefficients of the linear scheme (13) and where we have employed Lemma 2 in the last inequality. Making use of the assumption that $\Psi^{\prime}$ is bounded, we can control the nonlinear terms by the positive coefficients $b_{-1}, b_{0}$, and $b_{1}$ if $a$ is sufficiently small. We conclude

$$
S\left(k, h, n, i, V_{i}^{n+1}, V\right)-S\left(k, h, n, i, U_{i}^{n+1}, U\right) \geq 0,
$$

which completes the proof.

Acknowledgements. The authors acknowledge partial support from the EU Project "Hyperbolic and Kinetic Equations", grant HPRN-CT-2002-00282. The first and third author are partly supported by the the Deutsche Forschungsgemeinschaft, grants JU 359/3 (Gerhard-Hess Award) and JU 359/6 (Forschergruppe 518), and by the DAAD-Procope Program. The first author expresses his thanks to the Institute MIP of the University P. Sabatier in Toulouse, where a part of this research has been carried out, for the kind hospitality. 


\section{REFERENCES}

[1] G. Barles and B. Perthame, Discontinuous solutions of deterministic optimal stopping time problems. RAIRO Modél. Math. Anal. Numér. 21 (1987) 557-579.

[2] G. Barles and B. Perthame, Exit time problems in optimal control and vanishing viscosity method. SIAM J. Control Optim. 26 (1988) 1133-1148.

[3] G. Barles and H.M. Soner, Option pricing with transaction costs and a nonlinear Black-Scholes equation. Finance Stoch. 2 (1998) 369-397.

[4] G. Barles and P.E. Souganides, Convergence of approximation schemes for fully nonlinear second order equations. Asympt. Anal. 4 (1991) 271-283.

[5] G. Barles, Ch. Daher and M. Romano, Convergence of numerical schemes for parabolic equations arising in finance theory. Math. Models Meth. Appl. Sci. 5 (1995) 125-143.

[6] F. Black and M. Scholes, The pricing of options and corporate liabilities. J. Polit. Econ. 81 (1973) 637-659.

[7] R. Bodenmann and H.J. Schroll, Compact difference methods applied to initial-boundary value problems for mixed systems. Numer. Math. 73 (1996) 291-309.

[8] P. Boyle and T. Vorst, Option replication in discrete time with transaction costs. J. Finance 47 (1973) 271-293.

[9] G.M. Constantinides and T. Zariphopoulou, Bounds on process of contingent claims in an intertemporal economy with proportional transaction costs and general preferences. Finance Stoch. 3 (1999) 345-369.

[10] M. Crandall and P.L. Lions, Viscosity solutions of Hamilton-Jacobi equations. Trans. Amer. Math. Soc. 277 (1983) 1-42.

[11] M. Crandall, H. Ishii and P.L. Lions, User's guide to viscosity solutions of second order partial differential equations. Bull. Amer. Math. Soc. 27 (1992) 1-67.

[12] M. Davis, V. Panis and T. Zariphopoulou, European option pricing with transaction fees. SIAM J. Control Optim. 31 (1993) 470-493.

[13] B. Düring, M. Fournié and A. Jüngel, High order compact finite difference schemes for a nonlinear Black-Scholes equation. Int. J. Appl. Theor. Finance 6 (2003) 767-789.

[14] R. Frey, Perfect option hedging for a large trader. Finance Stoch. 2 (1998) 115-141.

[15] R. Frey, Market illiquidity as a source of model risk in dynamic hedging, in Model Risk, R. Gibson Ed., RISK Publications, London (2000).

[16] G. Genotte and H. Leland, Market liquidity, hedging and crashes. Amer. Econ. Rev. 80 (1990) 999-1021.

[17] S.D. Hodges and A. Neuberger, Optimal replication of contingent claims under transaction costs. Rev. Future Markets 8 (1989) $222-239$.

[18] V.P. Il'in, On high-order compact difference schemes. Russ. J. Numer. Anal. Math. Model. 15 (2000) 29-46.

[19] H. Ishii, A boundary value problem of the Dirichlet type for Hamilton-Jacobi equations. Ann. Scuola Norm. Sup. Pisa 16 (1989) 105-135.

[20] R. Jarrow, Market manipulation, bubbles, corners and short squeezes. J. Financial Quant. Anal. 27 (1992) 311-336.

[21] P. Kangro and R. Nicolaides, Far field boundary conditions for Black-Scholes equations. SIAM J. Numer. Anal. 38 (2000) $1357-1368$.

[22] D. Lamberton and B. Lapeyre, Introduction au calcul stochastique appliqué à la finance. $2^{\mathrm{e}}$ édn., Ellipses, Paris (1997).

[23] J. Leitner, Continuous time CAPM, price for risk and utility maximization, in Mathematical Finance. Workshop of the Mathematical Finance Research Project, Konstanz, Germany, M. Kohlmann et al. Eds., Birkhäuser, Basel (2001).

[24] R.C. Merton, Theory of rational option pricing. Bell J. Econ. Manag. Sci. 4 (1973) 141-183.

[25] D. Michelson, Convergence theorem for difference approximations of hyperbolic quasi-linear initial-boundary value problems. Math. Comput. 49 (1987) 445-459.

[26] E. Platen and M. Schweizer, On feedback effects from hedging derivatives. Math. Finance 8 (1998) 67-84.

[27] A. Rigal, High order difference schemes for unsteady one-dimensional diffusion-convection problems. J. Comp. Phys. 114 (1994) 59-76.

[28] P. Schönbucher and P. Wilmott, The feedback effect of hedging in illiquid markets. SIAM J. Appl. Math. 61 (2000) $232-272$.

[29] H.M. Soner, S.E. Shreve and J. Cvitanic, There is no nontrivial hedging portfolio for option pricing with transaction costs. Ann. Appl. Probab. 5 (1995) 327-355.

[30] G. Strang, Accurate partial difference methods. II: Non-linear problems. Numer. Math. 6 (1964) 37-46.

[31] C. Wang and J. Liu, Fourth order convergence of compact finite difference solver for 2D incompressible flow. Commun. Appl. Anal. 7 (2003) 171-191.

[32] A. Whalley and P. Wilmott, An asymptotic analysis of an optimal hedging model for option pricing with transaction costs. Math. Finance 7 (1997) 307-324. 